\title{
ADAPTIVE NEONATAL BRAIN SEGMENTATION: APPLICATION TO VENTRICULOMEGALY AND EXCESS EXTRA-AXIAL CEREBRAL-SPINAL FLUID
}

\author{
M.J. Cardoso ${ }^{1}$, A. Melbourne ${ }^{1}$, G.S. Kendall ${ }^{2}$, C. Hagmann $^{2}$, A. Bainbridge ${ }^{3}$, N. Marlow ${ }^{2}$, N.J. Robertson ${ }^{2}$, \\ S. Ourselin ${ }^{1}$ \\ ${ }^{1}$ Centre For Medical Image Computing, ${ }^{2}$ Academic Neonatology, University College London, ${ }^{3}$ Medical \\ Physics \& Bio-Engineering, University College London Hospital NHS Foundation Trust, London, UK
}

Background: Recent advances suggest that measurement of brain volumes in babies born prematurely can help define biomarkers for neurodevelopmental outcome. However, due to poor contrast, partial volume $(\mathrm{PV})$, and significant natural and pathological anatomical variability, techniques that measure brain volume are prone to error.

Methods: We propose an image segmentation that uses a maximum a posteriori Expectation Maximisation algorithm with novel adaptive priors on intensities and tissue proportions, with B0 inhomogeneity and spatial homogeneity correction terms. This robust technique enables the segmentation of images with high anatomical disparity from a normal population by iterative relaxation of priors toward the data. The proposed method implicitly models PV, mitigating the problem of poor GM/WM contrast.

Results: 43 T1-weighted volumes (1.5T Siemens Avanto) scanned at term equivalent age (mean gestational age $27.1 \pm 2.7$ weeks) were segmented. Two subsets were chosen for comparison to manual segmentation and a standard algorithm: 15 visibly normal neonates and 4 with anatomical distortion. Dice scores between manual segmentation and the proposed method were $0.908 \pm 0.034$ and $0.725 \pm 0.217$ for the standard method $(\mathrm{p}<0.01)$. On the second subset, the dice scores for the proposed method were $0.92,0.86,0.93$ and 0.94 , and $0.22,0.15,0.35$, and 0.55 for the standard method (fig1).

Conclusions: The proposed method allows significantly improved segmentation compared to a standard algorithm.

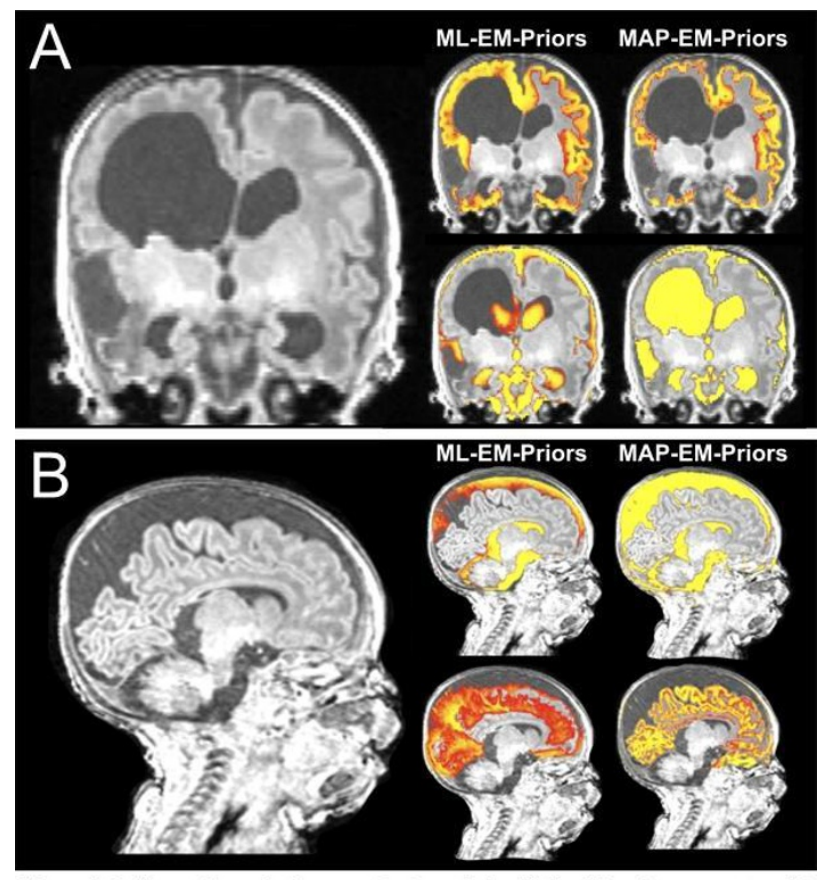

Figure 1: Subjects with marked asymmetrical ventricular dilation (A) and excess extra-axialCSF (B). Corresponding cortical GM and CSF segmentations with (ML-EM) and without (MAP[Figure1] 